Meta

Journal des traducteurs

Translators' Journal

\title{
Introduction de la notation phonologique pour la description du système notionnel
}

\section{Aniela Topulos}

Volume 32, numéro 4, décembre 1987

URI : https://id.erudit.org/iderudit/004496ar

DOI : https://doi.org/10.7202/004496ar

Aller au sommaire du numéro

Éditeur(s)

Les Presses de l'Université de Montréal

ISSN

0026-0452 (imprimé)

1492-1421 (numérique)

Découvrir la revue

Citer cet article

Topulos, A. (1987). Introduction de la notation phonologique pour la

description du système notionnel. Meta, 32(4), 442-448.

https://doi.org/10.7202/004496ar d'utilisation que vous pouvez consulter en ligne. 


\section{INTRODUCTION DE LA NOTATION TOPOLOGIQUE POUR LA DESCRIPTION DU SYSTĖME NOTIONNEL}

Les langues du monde entier se caractérisent par une grande diversité aussi bien dans l'ensemble des règles (grammaire) que dans celui des unités lexicales (terminologie). La traduction est néanmoins possible, ce qui témoigne de l'existence d'éléments communs. Au niveau de la forme (du son), l'on peut rechercher les propriétés communes des langues dans les capacités phonétiques et auditives de l'homme. Les propriétés physiologiques jouent donc un rôle décisif. Au niveau des significations, le trait commun des langues constitue les règles de génération des notions.

Les définitions de "la notion » sont nombreuses et suivant celle qu'on admet, il est possible de construire différentes théories se rapportant à la création du système notionnel.

Partant toutefois du principe que la notion est un cas particulier de la signification et que cette dernière constitue l'ensemble des équivalents intellectuels des traits, on peut établir une théorie cohérente, vérifiable pour chaque langue et rendant possibles, de façon précise, des recherches comparatives de différentes langues du point de vue sémantique. Les notions sont créées en tant que les quotients ou bien les sommes des équivalents intellectuels des traits.

L'homme apprend à connaître le monde par les sens qui enregistrent certaines propriétés du monde qui l'entoure. Les hommes, hormis quelques cas particuliers, sont munis des mêmes organes sensitifs, qui déterminent la connaissance du monde. Les sens décident aussi du système notionnel. Celui-ci permet à l'esprit humain de se créer un modèle du monde. Il rend possible non seulement la reproduction de ce monde perçu, mais aussi la construction des modèles des objets et des phénomènes qui ne trouvent pas d'équivalents dans la réalité qui entoure l'homme. Ce sont les règles de génération des notions qui en décident. Elles sont conformes sans aucun doute aux règles de la perception. Nous percevons un objet par l'ensemble de ses caractéristiques, nous sommes capables de distinguer ses traits particuliers, donc de discerner - indépendamment 
d'autres traits - la couleur de l'objet, son volume, sa dureté, son odeur, etc. Nous créons le modèle de cet objet dans notre esprit en tant qu'un ensemble d'équivalents intellectuels des traits et, en même temps, nous construisons le modèle de chacun de ces traits. Il en est de même avec les traits caractérisant ce que nous ressentons en notre for intérieur. Nous pouvons ensuite associer librement ces équivalents intellectuels des traits et obtenir ainsi des modèles des objets et des phénomènes, que nous n'avons jamais rencontrés dans la réalité qui nous entoure. Nous possédons une "imagination". Le manque de corrélation entre les langues prouve que la réalité présente diffère dans les différents points de notre globe et que cette réalité influence en grande partie l'activité créatrice de notre esprit. Au cours des années, entre les peuples, des divergences culturelles importantes sont nées, et nous pouvons retrouver leur reflet dans les systèmes notionnels différents pour les langues consécutives. Au sein de la même langue, nous pouvons également remarquer des particularités dans les systèmes notionnels de différents groupes de population et aussi des distinctions personnelles. Elles découlent d'un degré différent de la connaissance du monde, d'un autre type d'expérience ou de réflexions personnelles et des capacités diverses de perception (degré de la sensibilité des sens respectifs). Tout cela ne rend pas impossible pourtant l'échange de l'information entre les hommes qui proviennent aussi bien du même cercle culturel que de milieux différents. Il devient seulement un peu plus difficile. Ce fait témoigne de l'existence d'éléments communs pour toutes les langues, éléments qui décident de la possibilité de traduction des textes écrits dans des langues différentes. Ces éléments communs sont des équivalents intellectuels de la perception des traits et les règles de génération des notions. Elles sont les mêmes dans toutes les langues. Les divergences apparaissent au moment de l'application aux traits particuliers de valeurs définies, c'est-à-dire à l'évaluation des traits. Prenons par exemple le trait "couleur ". Dans différentes langues la distinction s'exprime par la gamme des couleurs. Il en est ainsi par suite des divergences existant dans le monde qui nous entoure (les Esquimaux par exemple distinguent plusieurs nuances du blanc, car cette couleur domine dans leur entourage) et des particularités culturelles qui sont nées au cours des siècles. Il en est de même avec d'autres traits caractéristiques. Comme une grande partie des équivalents intellectuels a été engendrée en tant que produit de nombreux traits simples, les différences dans les systèmes notionnels peuvent être importantes.

Pour démontrer les similitudes et les différences dans les systèmes notionnels, il est nécessaire d'utiliser un appareil adapté à la description qui permettra de caractériser ces sytèmes le plus fidèlement possible et de façon très précise. La langue naturelle permet évidemment de donner une caractéristique complète du système notionnel, mais les possibilités d'interprétation qu'il contient assombrissent l'image. Il est donc indispensable d'utiliser une langue adéquate qui ne permettra pas d'interpréter librement. Ce postulat peut être rempli grâce à la notation formelle, dont disposent les mathématiques.

Les linguistes tirent souvent profit de la notation de la théorie des ensembles qui pourrait être utilisée ici aussi. Pour représenter certains traits caractérisant les systèmes notionnels, la notation topologique est néanmoins plus efficace. Elle exige l'introduction de la notion de l'espace. Les mathématiques se servent souvent des notions d'ensemble et d'espace de manière interchangeable. Il existe pourtant des cas où l'on utilise uniquement la notion d'espace. Il en est ainsi quand l'ensemble étudié remplit des conditions déterminées. Le système notionnel constitue un ensemble de notions liées par des relations. Il est aussi soumis à certaines conditions. L'utilisation de la notation topologique pour la description du système notionnel exige tout d'abord que l'on prouve que l'ensemble des notions peut être considéré comme un espace topologique.

Prenons comme exemple l'ensemble des notions portant sur l'usinage. 
Usinage - Attribution à l'objet usiné de la forme, des dimensions et de la qualité de la surface requises, par l'élimination partielle de leur matériau à l'aide d'outils d'usinage.

À l'ensemble des notions portant sur l'usinage (noté par T) est attribuée une famille d'ensembles (c'est-à-dire un ensemble composé d'ensembles) dont la puissance égale le nombre des traits distingués qui caractérisent l'usinage. Prenons en considération quelques-uns des nombreux traits caractéristiques pour ce type de traitement (plus exactement quelques équivalents intellectuels des traits, puisque nous analysons des ensembles de notions).

1. Le type de la course de travail défini par rapport à l'allure de la trajectoire.

2. Le type du mouvement d'avance défini par rapport à l'allure de la trajectoire.

3. La périodicité de travail du tranchant.

4. La variation de la coupe de la couche usinée.

C'est un ensemble d'équivalents des traits qui, après s'être vu attribuer des valeurs correspondantes, engendrent des notions (ou plus précisément des ensembles de notions) se rapportant aux méthodes d'usinage respectives, à savoir : tournage, brochage, robotage, fraisage, forage.

Désignons successivement par $\mathbf{A}_{1}, \mathbf{A}_{2}, \mathbf{A}_{3}, \mathbf{A}_{4}$ les ensembles de notions distinguées à partir des quatre traits cités, et par la lettre $\mathrm{A}$ les familles composées de ces ensembles. L'étendue de chacun de ces ensembles est identique, c'est-à-dire chaque désignation de la notion représentée par l'appellation "usinage " est la désignation de chacun de ces ensembles. Les éléments de ces ensembles constituent les ensembles distingués à partir des intervalles de valeurs des traits respectifs (dans un cas particulier, ils peuvent constituer des ensembles à un seul élément). Les ensembles qui sont les éléments de l'ensemble $A_{1}$ (ou encore $A_{2}, A_{3}, A_{4}$ ) sont des ensembles disjoints, car par exemple l'ensemble des désignations de la notion représentée par l'appellation « usinage à course de travail rectiligne " est disjoint par rapport à l'ensemble des désignations de la notion représentée par l'appellation "usinage à course de travail curviligne", etc. Les ensembles constituant les éléments des ensembles $A_{2}, A_{3}, A_{4}$ se présentent d'une manière semblable.

Désignons par $R$ la famille des sous-ensembles distinguée dans l'ensemble $A_{1}$, à partir des intervalles des valeurs des traits. Ce seront les ensembles $\mathbf{R}_{1}, \mathbf{R}_{2}, \mathbf{R}_{\mathbf{3}} \ldots \mathbf{R}_{\mathbf{n}}$. Ils remplissent la condition suivante :

$\mathrm{UR}=\mathrm{UR}_{1}, \mathrm{UR}_{2} \mathrm{UR}_{3} \mathrm{U} \ldots \mathrm{UR}_{\mathrm{n}}=\mathrm{A}_{1}$, puisque tout ensemble des notions se rapportant à l'usinage, distingué à partir de l'intervalle des valeurs du trait "type de la course de travail défini par rapport à l'allure de la trajectoire " s'inclura dans l'ensemble distingué du point de vue de ce trait.

Il en résulte que la famille $R$ est le recouvrement de l'ensemble $A_{1}$.

L'étendue des ensembles $R_{1}, R_{2}, R_{3} \ldots R_{n}$ peut être divergente dans des langues différentes. L'étendue de la somme de ces ensembles est par contre indépendante de la langue. De même, l'on peut faire l'analyse des ensembles $\mathbf{A}_{2}, \mathbf{A}_{3}, \mathbf{A}_{4}$. Mais dans les cas où les ensembles de la famille $R$ sont disjoints (et cette disjonction est indispensable, si nous voulons ordonner la terminologie), la famille $\mathrm{R}$ est aussi une distribution de l'ensemble A. On peut constater ici que l'ordonnance de la terminologie du point de vue sémantique est la distinction, dans l'ensemble des notions donné que nous voulons ordonner, du trait attribuable à tous les éléments de cet ensemble, et ensuite la recherche de sous-ensembles tels qu'ils pourraient aussi bien constituer le recouvrement que la distribution de l'ensemble distingué du point de vue de ce trait.

Les notions de recouvrement et de distribution sont utilisées dans la théorie des ensembles et dans la topologie. 
Considérons encore une fois les ensembles de la famille A. Chacun de ces ensembles peut être ordonné de façon linéaire, tenant compte du trait qui a été à la base de la distinction de celui-ci. Tout trait est évalué d'une certaine manière, aussi bien dans le cas où son caractère est discret que continu (continuum). Ces valeurs peuvent être appliquées à l'ensemble des nombres naturels. Le trait " allure de la trajectoire du mouvement " a un caractère continu. L'étendue de la valeur s'inclut dans les limites partant de " la trajectoire rectiligne" passant par différentes sortes de "trajectoires curvilignes" jusqu'à « la trajectoire rotatoire ». Ce trait peut aussi être considéré comme discret, tenant compte seulement de ce type d'allures de la trajectoire qui sont définies par les appellations telles que par exemple "droite", "cycloïde", " hypocycloïde", " cercle". Toutefois, nous n'épuiserons pas alors toutes les allures possibles, le caractère discret du trait ne serait donc que l'effet d'une considérable simplification. Dans le cas d'un trait du genre de "périodicité de travail du tranchant ", il est possible de traiter l'ensemble appliqué à ses valeurs en tant que paire ordonnée $\langle 0,1\rangle$, soit le premier élément est la notion engendrée à la base de l'intervalle de valeur nulle du trait (dans notre cas, ce serait la "continuité ") - le second élément étant la " périodicité ". Quand nous supposons que la continuité est un cas particulier de la périodicité, nous reconnaissons que la "périodicité " est un trait qui caractérise le déroulement d'un phénomène par rapport au temps. Dans le second cas, la périodicité veut dire le trait susmentionné de valeur déterminée, c'est-à-dire le trait qui caractérise le déroulement d'un phénomène qui varie dans le temps par rapport à ce temps même. Il est indispensable de traiter les traits qui, dans la langue naturelle, sont reconnus être opposés en tant qu'un seul trait lorsqu'on veut décrire le phénomène avec précision. Remarquons qu'il y a un grand nombre de ces exemples, comme la droite qui est un cas particulier d'une courbe en mathématiques, la chaleur et le froid sont dans le langage de la physique de la chaleur, le beau et le mauvais temps en météorologie constituent le temps.

En considérant l'ensemble des valeurs du trait " périodicité " en tant que paire ordonnée dans l'ensemble " usinage " nous engendrons deux sous-ensembles, soit " usinage avec travail continu du tranchant" et "usinage avec travail cyclique du tranchant ". Ceci constitue, à l'évidence, une grande simplification, puisque nous savons que la périodicité, soit le déroulement du phénomène dans le temps, peut être variée. Il y a pourtant un grand nombre de situations où les deux intervalles présentés des valeurs du trait susmentionné sont tout à fait suffisants pour effectuer la description du phénomène, ou bien encore de l'objet. L'application des valeurs aux différents traits dépend des besoins et du degré de connaissance des éléments de la réalité déterminés qui caractérisent le trait donné. Ces valeurs peuvent toujours être ordonnées de manière qu'elles trouvent leur projection dans l'ensemble des nombres naturels. Cet ordre peut être varié. Dans la topologie, on distingue quatre types fondamentaux d'ordres, soit : 1) ordre dense, 2) ordre continu, 3) ordre lacunaire, 4) ordre par échelon. On peut rencontrer tous ces types d'ordres dans les systèmes notionnels. Le présent exposé n'a pas pour objectif de démontrer cette constatation.

Revenons à l'ensemble des notions ayant trait à l'usinage, soit à l'ensemble T. Sur cet ensemble la famille $A$ a été définie, ses éléments sont $A_{1}, \mathbf{A}_{2}, \mathbf{A}_{3}, \mathbf{A}_{4}$. Chaque ensemble de la famille $A$ est distingué sur la base du trait qui se rapporte à tous les éléments de l'ensemble $T$, c'est-à-dire que chaque sous-ensemble, soit $A_{1}, A_{2}, A_{3}, A_{4}$, est compris dans l'ensemble maximal du point de vue d'un trait déterminé, donc dans l'ensemble $T$. Les plus petits éléments de cet ensemble seront considérés comme des points. Chaque point de l'ensemble qui se caractérise par exemple par le trait 1 est inclus dans l'ensemble $T$, et il n'existe pas de point auquel se rapporterait le trait 1 qui ne ferait pas partie de l'ensemble T. Ainsi, chaque point de l'ensemble $T$ a un entourage inclus dans cet ensem- 
ble. Il en résulte que l'ensemble $T$ est ouvert. On caractérise de la même façon les ensembles $A_{1}, A_{2}, A_{3}, A_{4}$. Chaque point de ces ensembles a un entourage inclus dans ces ensembles, c'est-à-dire que chaque point de l'ensemble $A_{1}$ a un entourage inclus dans l'ensemble $A_{1}$, etc. Ces ensembles, comme on l'a déjà dit, ont des étendues identiques à celles de l'ensemble T. Il peut être démontré aussi que ces ensembles sont aussi des ensembles fermés (puisque leur complément est un ensemble vide).

Il faut remarquer que les ensembles de la famille A, à laquelle appartient aussi tout l'ensemble $\mathrm{T}$, sont indépendants de la langue. Ils seront identiques dans les systèmes notionnels dans chacune des langues existantes à condition, évidemment, que dans la langue donnée l'usinage soit décrit et caractérisé du point de vue des traits cités plus haut. Le choix des traits caractéristiques est indépendant de la langue et est déterminé par la théorie se rapportant à une méthode définie, à un phénomène, etc.

Les ensembles distingués sur les ensembles $A_{1}, A_{2}, A_{3}, A_{4}$, soit les ensembles cités comme exemple dans la famille $\mathbf{R}$, ont un caractère différent. Ils ont été engendrés sur la base des intervalles des valeurs des traits. Comme il a déjà été souligné, dans différentes langues des "systèmes de mesure des traits " différents se sont créés de façon naturelle. C'est pourquoi les ensembles de la famille $\mathbf{R}$ peuvent avoir des étendues différentes dans les langues différentes, seule l'étendue de la somme de ces ensembles sera indépendante de la langue.

Considérons ces ensembles et répondons à la question : sont-ils ouverts ou fermés ?

Dans les systèmes de langue naturels, où les intervalles des valeurs des traits sont considérés comme des traits séparés, ces ensembles sont ouverts. Cela veut dire que dans le système de langue naturel, les ensembles de notions désignés par des appellations " usinage avec course de travail curviligne " et " usinage avec course de travail rectiligne " sont des ensembles disjoints et n'ont pas de limite commune. Ces notions, engendrées de cette manière, perdent leur utilité lorsque la trajectoire du mouvement éveille le doute quant à son allure rectiligne ou curviligne. Dans de tels cas, la langue demande un traitement artificiel, c'est-à-dire la détermination d'une limite arbitraire qui divise les trajectoires rectilignes et curvilignes, il faut donc définir quelle grandeur d'écart de la droite décide du fait qu'une trajectoire est appelée " curviligne ". En introduisant la langue topologique, nous allons effectuer l'opération de fermeture. Ce genre d'opération est souvent utilisé dans la terminologie, bien que cela ne soit pas toujours fait de façon correcte. Il est facile de le démontrer en s'appuyant sur l'exemple des ondes électromagnétiques radioélectriques.

micro-ondes - ondes électromagnétiques de 1 à $10 \mathrm{~m}$ de longueur ondes courtes - ondes électromagnétiques de 10 à $100 \mathrm{~m}$ de longueur petites ondes - ondes électromagnétiques de 100 à $1000 \mathrm{~m}$ de longueur grandes ondes - ondes électromagnétiques de 1000 à $30000 \mathrm{~m}$ de longueur

On a introduit ici un ordre qui peut éveiller un certain scepticisme, car le dernier élément de la première classe /micro-ondes/ est à la fois le premier élément de la seconde classe /ondes courtes/, etc. On obtient de la sorte des ensembles non disjoints, bien que l'objectif de cette opération fût d'obtenir des ensembles disjoints. On a effectué ici l'opération de fermeture de deux côtés de chaque ensemble. Si l'on veut toutefois obtenir des ensembles disjoints, cette opération doit être effectuée d'un seul côté, soit chaque classe (ensemble) doit avoir un élément premier ou un élément dernier, elle ne peut posséder par contre un élément premier et un élément dernier. Appelons $\mathbf{x}$ la longueur d'onde ; lorsque l'opération de fermeture est effectuée régulièrement, on obtient : 
les micro-ondes se caractérisent par $1 \mathrm{~m}<\mathrm{x} \geqslant 10 \mathrm{~m}$, les ondes-courtes :

les petites ondes :

$10 \mathrm{~m}<\mathrm{x} \geqslant 100 \mathrm{~m}$

les grandes ondes:

$100 \mathrm{~m}<\mathrm{x} \geqslant 1000 \mathrm{~m}$

$1000 \mathrm{~m}<\mathrm{x} \geqslant 30000 \mathrm{~m}$.

On obtient de la sorte des ensembles fermés à gauche. Cette opération peut être effectuée de façon à obtenir des ensemble fermés à droite. Mais ce qui a toujours lieu c'est que ces ensembles sont toujours fermés d'un côté. Effectuant ce genre d'opération de fermeture des ensembles, on a établi un ordre dense.

L'opération de fermeture devrait être une activité fondamentale de normalisation.

Indépendamment du fait qu'on effectue ou non l'opération de fermeture, les ensembles de la famille $A$ déterminée sur l'ensemble $T$, tout comme la famille $R$ définie sur l'ensemble $A_{1}$, remplissent les conditions suivantes si l'on fait certaines hypothèses : $A_{1} \cup A_{2} \cup A_{3} \cup A_{4} \quad A$

La somme des ensembles de la famille $A$ est un élément de la famille $A, c$ 'est-àdire de la famille définie sur l'ensemble $T$, tout comme

$$
\mathbf{R}_{1} \cup \mathbf{R}_{2} \cup \mathbf{R}_{3} \cup \mathbf{R}_{4} \quad \mathbf{R}
$$

La somme des ensembles de la famille $R$ est un élément de la famille $R$, c'est-àdire un élément de la famille des ensembles définie sur l'ensemble $A_{1}$,

$$
A_{1} \cap A_{2} \cap A_{3} \cap A_{4} \quad A
$$

la multiplication des ensembles de la famille $A$ est un élément de la famille $A$, tout comme

$$
\mathbf{R}_{1} \cap \mathbf{R}_{2} \cap \mathbf{R}_{3} \cap \mathbf{R}_{\mathbf{4}} \quad \mathbf{R}
$$

la multiplication des ensembles de la famille $R$ est un élément de la famille $R$.

Un ensemble vide est un élément de la famille $A$ et un élément de la famille $R$. L'ensemble $T$ est un élément de la famille $A$, et l'ensemble $A_{1}$ un élément de la famille R.

Ce genre de conditions est imposé à l'espace topologique. Elles peuvent donc être imposées aux ensembles du système notionnel. Les ensembles de ce système peuvent dont être traités et décrits comme l'espace topologique. Les ensembles peuvent être ouverts, fermés ou ouverts-fermés.

Une topologie type qui peut servir à décrire les ensembles des systèmes notionnels est la topologie de Tichnonow, soit la topologie qui est la projection de la multiplication cartésienne dans l'espace topologique. Tenant compte de l'exemple donné de l'ensemble des notions se rapportant à l'usinage, l'on peut admettre que les quatre traits fondamentaux cités ici qui caractérisent cet ensemble (ce sont aussi des traits typiques de classification) sont quatre coordonnées, et à chacune d'elles peut être attribuée une valeur définie. Comme résultat des multiplications des traits et des valeurs définies l'on obtient des ensembles de notions qu'on désigne par les noms "tournage", " forage ", " rabotage", "brochage ", " fraisage ", soit des ensembles de notions se rapportant aux méthodes fondamentales d'usinage.

Les points de cet espace sont des multiplications de traits de valeur unitaire d'intervalle. Ainsi, le nombre de points de cet espace dépend du nombre d'intervalles déterminés des valeurs unitaires du trait.

Dans le cas de traits de caractère continu, les points de cet espace peuvent être déterminés de façon analogique à celle des points de l'espace métrique. Ils n'ont pas de dimensions et leur nombre peut être infini. Entre les points de cet espace et l'ensemble des termes il n'y a pas de correspondance univoque, c'est-à-dire que dans le cas où l'un (ou nombre) des traits qui constituent les coordonnées de cet espace est un trait continu, à de nombreux points de cet espace correspond un seul terme. 
Dans les ensembles de notions traités en tant qu'espace topologique on peut aussi distinguer une sous-base et une base.

Les ensembles de la famille A constitueront la sous-base de l'espace susdéfini ; la base, par contre, sera constituée par les ensembles qui sont les multiplications des ensembles $\mathbf{A}_{1} \cap \mathbf{A}_{2} \cap \mathbf{A}_{3} \cap \mathbf{A}_{4}$.

La sous-base est indépendante de la langue; la base, elle, est différente dans des langues différentes, car elle dépend du degré d'évaluation des traits consécutifs et de la distinction des sous-ensembles dans les ensembles $A_{1}, A_{2}, A_{3}, A_{4}$.

Comme il ressort de ce qui vient d'être dit, pour effectuer l'unification des systèmes notionnels il faut adopter des systèmes de mesure de valeurs des traits semblables. Au fur et à mesure que les notions se rapprochent les unes des autres, ce processus s'approfondit de plus en plus. Cela a lieu en particulier dans les langues des disciplines qui demandent une coopération plus étroite. Les différences dans les systèmes notionnels constituent toutefois un certain obstacle quant à l'implantation de noms étrangers, car ils portent avec eux souvent une notion qui trouble le système en usage jusqu'à présent (il demande un autre moyen d'évaluation des traits) et c'est par là que l'on peut expliquer l'aversion fréquente pour une terminologie étrangère. L'acceptation d'une nouvelle théorie demande l'acceptation des éléments qui la constituent, soit de l'ensemble des notions, ou encore l'ajustement d'un autre ensemble de notions qui permettent de comprendre cette théorie. Cette seconde opération déforme souvent la théorie même. L'implantation d'une terminologie étrangère, lors de l'intraduction d'une nouvelle notion, n'appauvrit pas la langue, ce que redoutent tellement les puristes, mais elle l'enrichit, et il ne s'agit pas ici de richesse dans le sens d'un nombre plus grand de mots, mais de la richesse d'un système notionnel qui lui, de sa part, décide de nos moyens intellectuels. Mais cet enrichissement toutefois ne s'effectue pas sans symptômes négatifs qui sont la superposition de notions de différents systèmes, ce qui entraîne l'incompréhension et une fausse interprétation, et fait que l'appareil notionnel devient moins effectif. Il est donc indispensable d'effectuer l'analyse des systèmes notionnels, de souligner les différences et d'ordonner sciemment. L'utilisation de la notation topologique pour la description des systèmes notionnels permet de déterminer monosémiquement les irrégularités d'un système donné, ainsi que les analogies et les différences des systèmes qui correspondent dans des langues différentes. 